\title{
Real-World Experience Using Topical Therapy- Calcipotriol and Betamethasone Dipropionate Foam in Adults with Beyond-Mild Psoriasis
}

\author{
Roland Aschoff · Antonio Martorell · Tobias Anger • Diane Chayer • \\ Anthony Bewley
}

Received: December 18, 2020 / Accepted: February 4, 2021 / Published online: March 15, 2021

(C) The Author(s) 2021

\section{ABSTRACT}

Introduction: Determining optimal treatment for moderate plaque psoriasis can be challenging. Recent studies have demonstrated the effectiveness of calcipotriol and betamethasone dipropionate (Cal/BD) foam in patients with moderate-to-severe plaque psoriasis.

Methods: This research explored the use of Cal/BD foam in patients with beyond-mild psoriasis (defined as patients eligible for topical treatment, systemic treatment, or combined topical/systemic treatments) using an online questionnaire, retrospective medical-record review, and by capturing the attitudes and

Supplementary Information The online version contains supplementary material available at https:// doi.org/10.1007/s13555-021-00501-3.

\section{R. Aschoff}

Department of Dermatology, Faculty of Medicine and University Hospital Carl Gustav Carus,

Technische Universität Dresden, Dresden, Germany

A. Martorell ( $\square)$

Dermatology and Venereology Service, Hospital de

Manises, Valencia, Spain

e-mail: amartorell@hospitalmanises.es

T. Anger · D. Chayer

LEO Pharma A/S, Ballerup, Denmark

A. Bewley

Dermatology Department, Barts Health NHS Trust

and Queen Mary University, London, UK treatment approaches of dermatology specialists.

Results: Data from 409 patients with beyondmild psoriasis treated with Cal/BD foam were provided by 120 dermatology specialists from Germany, Spain, and the UK. Cal/BD foam was prescribed as monotherapy for most (58\%) patients and was considered to be effective. $\mathrm{Cal} / \mathrm{BD}$ foam was infrequently used in combination with biologics (7\%) in this segment; $26 \%$ of patients received Cal/BD foam in combination with a non-biologic systemic treatment. Cal/BD foam prescribers generally use topical agents to bridge the waiting time to non-biologic/biologic systemic treatment, and as an add-on to systemic treatment for residual lesions.

Conclusions: In patients with beyond-mild psoriasis, the most commonly prescribed topical medication was Cal/BD foam, which was regarded as an effective treatment. Further research is needed to determine optimal use of $\mathrm{Cal} / \mathrm{BD}$ foam in these patients.

Keywords: Calcipotriol/betamethasone dipropionate aerosol foam; Real-world experience; Systemic therapy; Topical therapy 


\section{Key Summary Points}

\section{Why carry out this study?}

Determining optimal treatment for moderate plaque psoriasis can be challenging.

While there are clear treatment pathways for mild and severe psoriasis, there exists a gray area between these two ends of the spectrum where optimal patient management is uncertain.

This research explores the use of calcipotriol and betamethasone dipropionate (Cal/BD) foam in patients within this gray area using an online questionnaire, retrospective medicalrecord review, and by capturing the attitudes and treatment approaches of dermatology specialists.

\section{What was learned from the study?}

Within the study population, Cal/BD foam was prescribed as monotherapy for (58\%) patients and was considered to be effective.

Prescribers also used topical agents to bridge the waiting time to non-biologic/ biologic systemic treatment, and as an add-on to systemic treatment for residual lesions.

Healthcare professionals in this research believed that the availability of increasingly efficacious topical treatments has widened the pool of patients eligible for such treatment. Giving patients the choice of an alternative treatment for psoriasis supports a "patient-centered" approach, which may enhance treatment adherence.

\section{DIGITAL FEATURES}

This article is published with digital features, including a summary slide, to facilitate understanding of the article. To view digital features for this article go to https://doi.org/10.6084/ m9.figshare.13705378.

\section{INTRODUCTION}

Psoriasis is a systemic inflammatory disease primarily involving skin and joints affecting up to $4 \%$ of the European population [1], most of whom present with chronic plaque psoriasis [2]. Psoriasis can profoundly impact patient quality of life (QoL), with patients often experiencing bothersome physical symptoms and significant psychosocial burden $[3,4]$. Despite the considerable prevalence and burden of plaque psoriasis, determining the most appropriate treatment for patients-especially those with moderate psoriasis-can be challenging. While there are clear treatment pathways for mild and severe psoriasis, there exists a gray area between these two ends of the spectrum where optimal patient management is uncertain. This could be because of variation in treatment guidelines relating to when systemic therapies should be started, and variations in the definition of "moderate" plaque psoriasis [5-10].

Most treatment guidelines recommend topical therapies for mild and mild-to-moderate psoriasis, either as monotherapy or as adjunctive therapy where psoriasis is not fully controlled by other treatments [5-10]. When used as an adjunct, topical therapies may either be given as multi-therapy (multiple topical therapies, normally used on different body locations) or as adjunctive therapy (with systemic treatments). Systemic treatments are recommended for use in patients with moderate-to-severe or severe psoriasis [5-10]. Given the overlap in guidelines for moderate plaque psoriasis, patients in this group may be eligible for either topical and/or systemic treatment.

The American Academy of Dermatology classifies moderate psoriasis as that which affects from at least $5 \%$ to less than $10 \%$ of body surface area (BSA), while European guidelines 
classify moderate-to-severe psoriasis as that which affects more than $10 \%$ of BSA $[5,9]$. However, additional factors can affect disease severity and its classification. For example, psoriasis can be more severe when sensitive body sites, such as face, genitals, and scalp, are involved $[10,11]$. These limitations in classification and unclear definition of moderate plaque psoriasis result in considerable variation in the treatment decisions of specialists in dermatology.

Topical therapies for psoriasis have evolved over time, reflecting advances in the understanding of disease pathogenesis [12], but there is sparse information on the potential effectiveness of these agents in moderate or moderate-severe psoriasis. Among newer topical therapies is calcipotriol and betamethasone dipropionate (Cal/BD) foam, approved for the treatment of plaque psoriasis in adults. It is a fixed-dose formulation of $\mathrm{Cal} / \mathrm{BD} \quad 50 \mu \mathrm{g} / \mathrm{g}$ $+0.5 \mathrm{mg} / \mathrm{g}$ cutaneous foam (Enstilar ${ }^{\circledR}$, LEO Pharma A/S, Denmark), applied once daily [13]. Studies have demonstrated superior efficacy of $\mathrm{Cal} / \mathrm{BD}$ foam in the treatment of plaque psoriasis over fixed-combination formulation gel and ointment, as well as over the individual active ingredients of the foam base [14-16].

We aim to investigate data from patients eligible for topical treatment, systemic treatment, or combined topical/systemic treatments, excluding patients with mild psoriasis where guidelines are clear. This population includes mainly patients with moderate psoriasis, but also some patients with severe psoriasis as they may benefit from add-on topical therapy (see Table 1 for patient selection criteria). To describe this population, the expression "beyond-mild" psoriasis was defined by three psoriasis specialists, in collaboration with LEO Pharma and a market research company (Cello Health Insight, London, UK), following a review of the academic literature. The literature review selected studies with the following characteristics: patients with moderate to severe disease; more than 50 patients; treatment either available or with potential to be licensed by the European Medicines Agency. More weight was given to studies with an active comparator (not
Table 1 Criteria for patient chart selection

\section{Characteristics}

$\geq 18$ years of age

Diagnosed with chronic plaque psoriasis and no other forms of psoriasis present. Affected areas must have included trunk and/or limbs

Under the respondent's current direct care

Not currently part of a clinical trial

Initiated $^{\mathrm{a}}$ on $\mathrm{Cal} / \mathrm{BD}$ foam (solely, or in addition to, another topical or systemic [biologic/non-biologic]) treatment by the HCP and had a follow-up appointment 1-3 months later

Could have been treatment-naïve before initiation of $\mathrm{Cal} / \mathrm{BD}$ foam

Eligible for either a topical, systemic (biologic/nonbiologic) treatment, or combined topical/systemic treatment regimen at the point of initiating $\mathrm{Cal} / \mathrm{BD}$ foam

If received topical steroid monotherapy prior to $\mathrm{Cal} / \mathrm{BD}$ foam, must have required additional treatment to topical steroid monotherapy to control psoriasis

If received systemic/phototherapy/biologic therapy prior to Cal/BD foam, must have had no response or partial response only (defined as $<75 \%$ change in PASI)

If measured, PASI $\geq 6$ and $\leq 20$; BSA $\geq 3 \%$

and $\leq 30 \%$; PGA $>1$ (or not "clear")

$B S A$ body surface area, $C a l / B D$ calcipotriol/betamethasone dipropionate, $H C P$ healthcare professional, $P A S I$ Psoriasis Area and Severity Index, $P G A$ Physician Global Assessment

a "Initiated" refers to the first instance where Cal/BD foam was received by the patient (this can be in addition to another therapy)

placebo/vehicle), and informative severity measures and outcomes.

In addition, we aim to investigate opinions from dermatology healthcare professional (HCP) specialists to understand current treatment regimens and specialist opinions relating 
to the use of Cal/BD foam as a treatment option for these patients.

\section{METHODS}

\section{Research Design}

In the retrospective, observational review of medical records at clinical practices in Germany, Spain, and the UK, participating specialists (dermatologists and general practitioners with a special interest [GPwSI] in dermatology) were asked to complete four anonymized patient record forms (PRFs) via a 40-min online selfcompletion questionnaire. Their general attitudes and treatment approaches regarding beyond-mild psoriasis were also captured using the online questionnaire. As such, specialists provided data on three patient subgroups: (1) beyond-mild patients treated with $\mathrm{Cal} / \mathrm{BD}$ foam; (2) the beyond-mild patient pool, which referred to the caseload of patients who met the beyondmild criteria; and (3) the wider patient pool, which referred to all patients with plaque psoriasis, regardless of severity.

The primary objective of this research was to investigate data from patients with beyondmild psoriasis, together with opinions from dermatology HCP specialists to understand current treatment regimens and specialist opinions relating to the use of $\mathrm{Cal} / \mathrm{BD}$ foam as a treatment option for these patients and to test the following hypotheses:

1. Cal/BD foam is currently used as a monotherapy in the beyond-mild setting.

2. Cal/BD foam is currently used in combination therapy with biologic systemic treatments.

3. Specialists express a preference for $\mathrm{Cal} / \mathrm{BD}$ foam in specific patient subpopulations.

4. Cal/BD foam can prolong the use of topical treatment before systemic treatment.

\section{Healthcare Professionals}

Dermatologists and GPwSI in dermatology who treated patients with plaque psoriasis and who wrote at least 10 prescriptions for Cal/BD foam within the last 3 months for patients in the beyond-mild psoriasis segment were invited to participate in this research. The specialists had to be responsible for making decisions regarding psoriasis treatment and follow-up care and have been in practice for more than 3 years and less than 30 years, with $70 \%$ or more of their time spent providing direct medical patient care.

Participating specialists were identified using online panels and a supplementary telephone participatory invitation. A purposive sampling method was used to achieve a broadly representative sample of specialists and of the patients they treat.

\section{Patient Data}

Participating specialists were asked to review the medical records of patients (aged at least 18 years) with beyond-mild plaque psoriasis, under their direct care, who were prescribed Cal/BD foam (as monotherapy or as an adjunct to another topical or systemic therapy), and who had at least one follow-up appointment within 1-3 months after initiation.

\section{Criteria for Patient Chart Selection}

Patients with beyond-mild psoriasis were defined as those eligible for either topical, systemic, or combined topical/systemic treatment and fulfilling 10 selection characteristics (Table 1). Patients with Psoriasis Area and Severity Index (PASI) $>20$ or BSA $>30 \%$, and patients receiving systemics (biologic/nonbiologic) as monotherapy, were excluded.

\section{Ethical Conduct of the Questionnaire}

The names and other identifying information about the specialists and the patient data they provided were confidential, and not shared with the study sponsor. Informed consent was obtained from all HCPs, and the research was conducted in accordance with guidelines/codes of conduct regarding anonymity and confidentiality from the Association of the British Pharmaceutical Industry, the European 
Pharmaceutical Market Research Association, the European Society for Opinion and Marketing Research, the Market Research Society, the British Healthcare Business Intelligence Association, and the European General Data Protection Regulation. Specialists were also informed about the compensation for their time, that all patient cases were anonymized, and that only adults were to be included. As all patient cases were anonymized, no personal data were retrospectively collected from available patient charts, and no written informed consent was required from patients. As such, approval by an ethics committee or institutional review board was not required.

\section{Data Collection}

Data were collected using structured questionnaires in English, Spanish, and German. The questionnaires used data-gathering methodology that involved a standard set of questions asked in the same manner and order, providing appropriate question flow and logic, based on responses provided. Of note, local rules related to market research resulted in a lower number of PRFs of patients receiving Cal/BD foam in the UK.

\section{Statistical Analyses}

Data were collected between 25 March 2019 and 22 April 2019, with the final data analyses performed in May 2019. All data were analyzed descriptively, and significance testing was performed at a confidence interval of 95\% ( $z$ test for two proportions and $t$ test for two means). Testing assumed a non-overlapping sample; therefore, results were not theoretical for a sample with an overlapping sample. Throughout the fieldwork and prior to conducting the analysis, the quality of data was checked to identify possible data entry errors.

\section{RESULTS}

A total of 120 specialists from clinical practices in Germany, Spain, and the UK completed the
Table 2 HCP participation: clinical practices in Germany, Spain, and the UK

Germany
$\begin{aligned} & \text { GPs with a } \\ & \text { special }\end{aligned}$
interest in
dermatology

online questionnaire (Table 2). A minority of dermatologists (13\%) and GPwSI (26\%) were not invited to participate since they had not met the criteria related to prescription of $\mathrm{Cal} / \mathrm{BD}$ foam to at least 10 beyond-mild patients in the past 3 months (Table $S 1$ in the supplementary material).

The most common age of the specialists interviewed was between 36 and 45 years (46\% in the UK, $46 \%$ in Spain, and 43\% in Germany), and the mean length of time they had been practicing was 11.6 years (10.2 years in the UK, 11.0 years in Spain, and 13.6 years in Germany).

Overall, $409 \mathrm{Cal} / \mathrm{BD}$ foam PRFs were completed, consisting of 89 PRFs (22\%) from the UK and 160 PRFs (39\%) each from Spain and Germany. On average, nearly two-thirds (64\%) of the clinical caseload included patients with beyond-mild psoriasis (UK, 77\%; Spain, 58\%; and Germany, 57\%). Over $60 \%$ of patients in this research had psoriasis that affected either their elbows (69\%), arms (66\%), legs $(64 \%)$, or back (60\%). 


\section{Current Topical Use in Beyond-Mild Psoriasis}

Almost all the specialists found it easy to identify beyond-mild psoriasis (89\% selected either "somewhat agree", "agree", or "strongly agree"). Most specialists also stated that they prescribed according to treatment guidelines for this patient group (82\% selected either "somewhat agree", "agree", or "strongly agree").

Topical therapy was the most prescribed treatment in beyond-mild patients $(58 \%$ of patients), followed by non-biologic systemic therapy (42\%) and biologic therapy (36\%). Topical therapy was frequently used as an adjunct to increase efficacy of non-biologic systemic therapy $(70 \%$ of specialists do so "often" or "very often"), but less frequently to increase efficacy of biologic treatment (52\%). Topical therapy was also frequently prescribed to bridge the waiting time until it is planned for a patient to receive systemic therapy (non-biologics, 64\%; biologics, 63\%).
The most prescribed topical therapy for beyond-mild patients was Cal/BD foam; its use was higher in beyond-mild patients (46\%) than the wider patient pool (37\%; $p=0.007$ ) (Fig. 1). The next most commonly prescribed topical therapy was Cal/BD gel $(21 \%$ and $17 \%$ of patients, respectively; $p=0.095$ ). Most patients who were started on Cal/BD foam had a PASI score of 10 or 11 (73\% of the PRFs had a PASI recorded, and the mean PASI score was 10), though patients did span the full range of the beyond-mild PASI criteria (6-20).

\section{Cal/BD Foam as a Monotherapy in the Beyond-Mild Setting}

The use of Cal/BD foam as monotherapy in beyond-mild patients was high, as demonstrated through the PRFs of patients treated with Cal/BD foam (58\% [236/409 patient cases]). When $\mathrm{Cal} / \mathrm{BD}$ foam was used as monotherapy, the patients tended to be younger (mean age 39 vs. 46 years; $p<0.001$; Fig. 2a) and more recently diagnosed than when

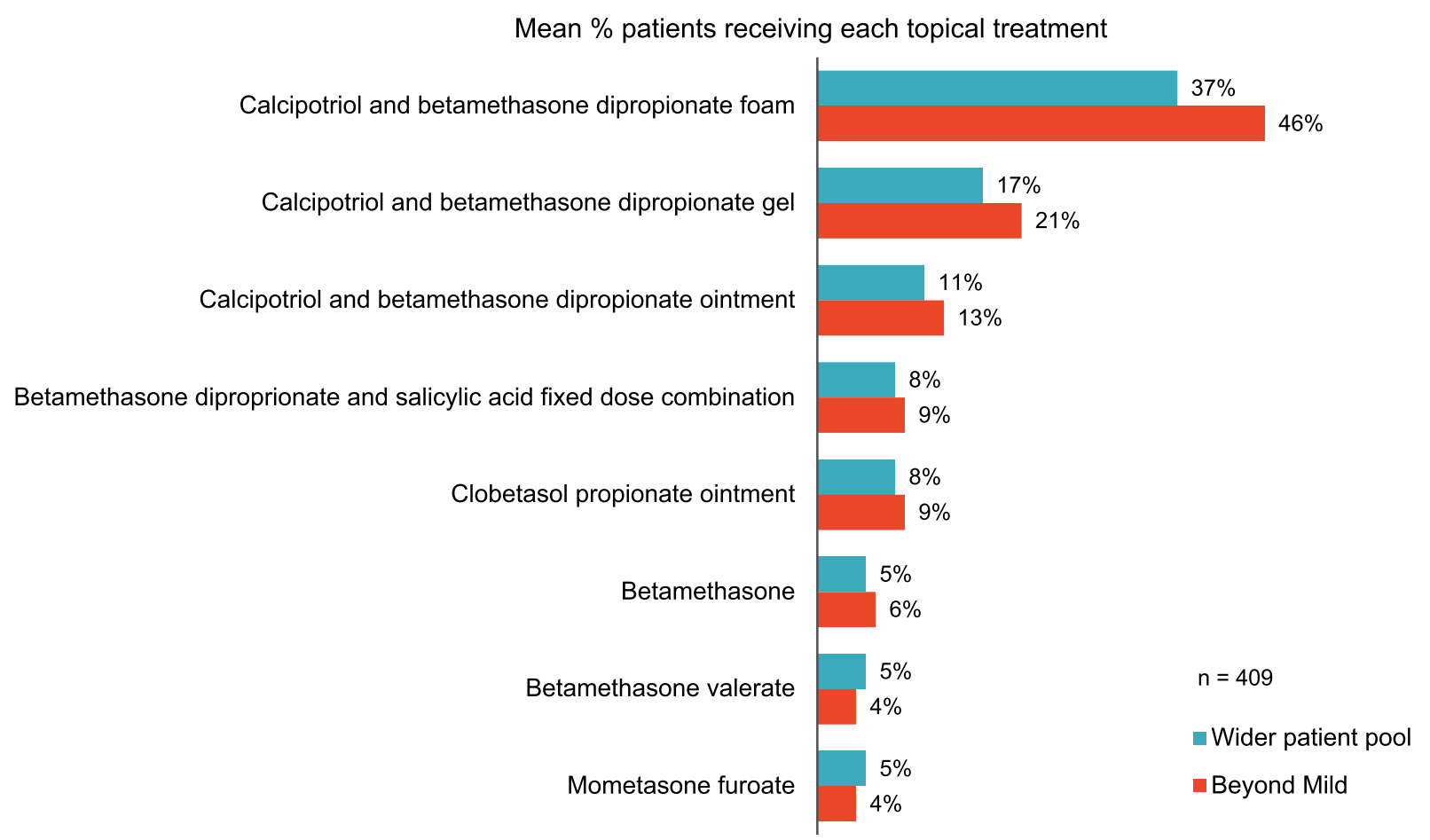

Fig. 1 Use of topical therapies (monotherapy or multiple) for plaque psoriasis. Only products accounting for at least $4 \%$ of topical treatments are shown 
(a)

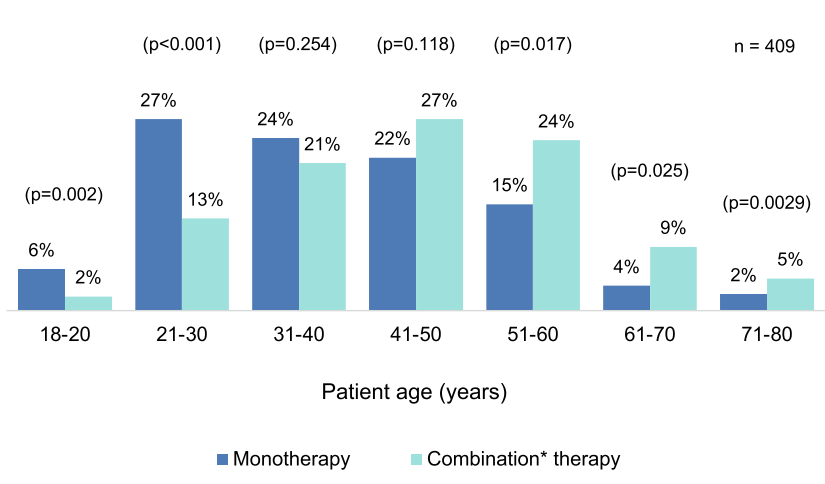

(b)

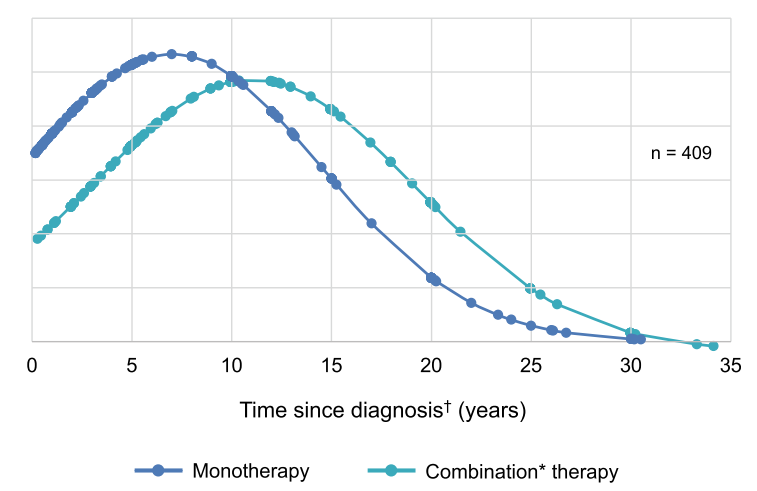

(c)

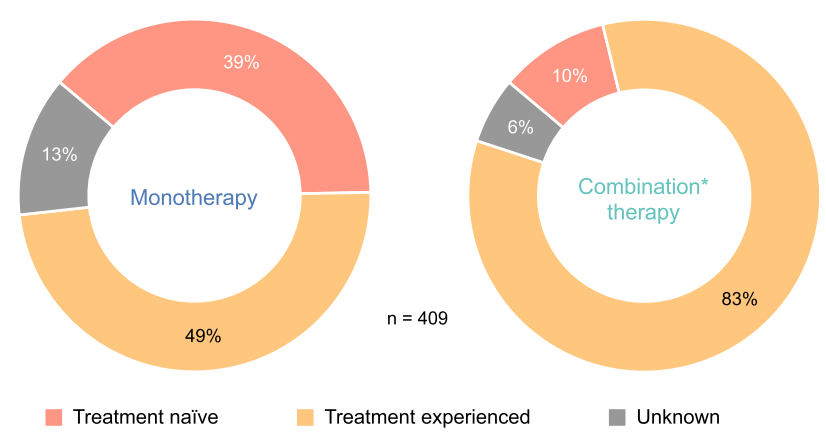

Fig. $2 \mathrm{Cal} / \mathrm{BD}$ foam used as monotherapy versus combination therapy: selected patient characteristics as based on patient record forms. a Patient age; $\mathbf{b}$ time since diagnosis; c treatment experience. ${ }^{*}$ “Combination” refers to patients on multiple topical therapy with $\mathrm{Cal} / \mathrm{BD}$ foam or receiving more than one type of treatment (e.g., topical + biologic). Multiple topical therapy treatments are not necessarily used on the same area. ${ }^{\dagger}$ Outliers removed used in combination therapy (mean time since diagnosis 7 vs. 11 years; Fig. 2b). Patients treated with Cal/BD foam monotherapy versus combination therapy were also more likely to have less severe psoriasis as measured by PASI $(10 \%$ vs. $12 \% ; p<0.001)$ and to have been treatmentnaïve at initiation of Cal/BD foam (39\% vs. 10\%; $p<0.001$; Fig. 2c) compared with those on adjunctive therapy. Specialists used Cal/BD foam in all lines of therapy; its use was not limited to second- or later-line treatment.

\section{Use of Cal/BD Foam in Combination with Biologic Systemic Treatments}

Only $7 \%$ of patients on Cal/BD foam were using it in combination with biologic systemic treatments; however, if an adjunctive topical agent was chosen, Cal/BD foam was the most commonly prescribed for beyond-mild patients (68\% of HCPs). When used as an adjunctive therapy, Cal/BD foam was mostly used with non-biologic systemic agents $(26 \%$ of patients on Cal/BD foam). For $42 \%(173 / 409)$ of beyondmild patients treated with $\mathrm{Cal} / \mathrm{BD}$ foam, it is either as part of multi-therapy (i.e., the use of multiple topical treatments normally used on different body locations) or as an adjunct to systemic therapies (some patients may have been on multiple therapies, receiving more than one type of additional treatment to Cal/BD foam). The non-biologic systemic therapy with which Cal/BD foam was most commonly used as an adjunct was methotrexate in the UK and Spain ( $16 \%$ and $10 \%$ of cases, respectively), and dimethyl fumarate in Germany (11\% of cases) (Fig. 3). 
(a)

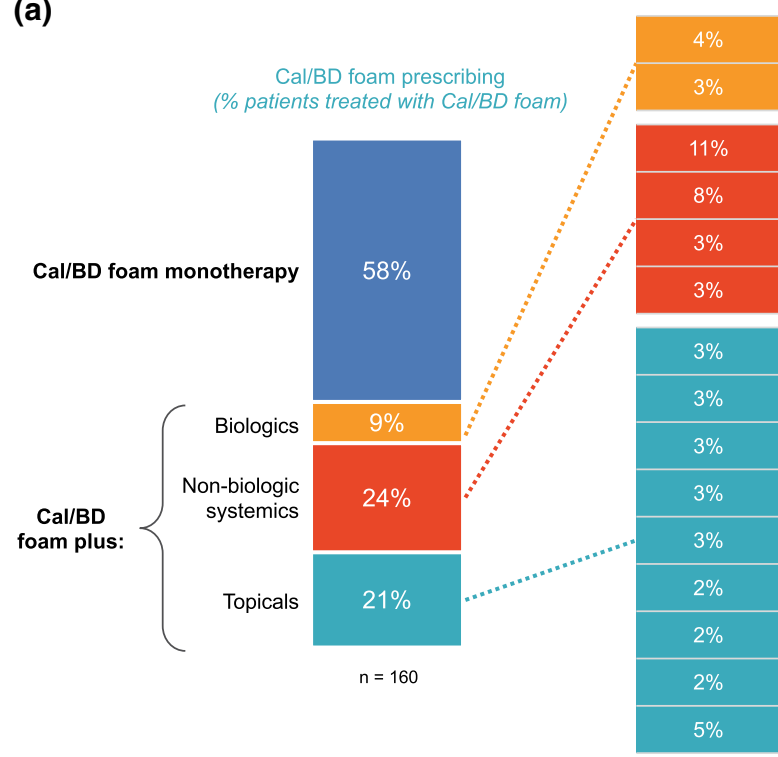

Anti IL-17/IL 17R

Anti IL-23/Anti IL-12

Dimethyl fumarate

Phototherapy

Apremilast

Methotrexate

Calcipotriol and betamethasone dipropionate gel

Mometasone

Calcipotriol and betamethasone dipropionate ointment

Calcipotriol and betamethasone dipropionate

Betamethasone dipropionate

Betamethasone

Clobetasol propionate ointment

Betamethasone valerate

Other prescribed topical treatments (b)

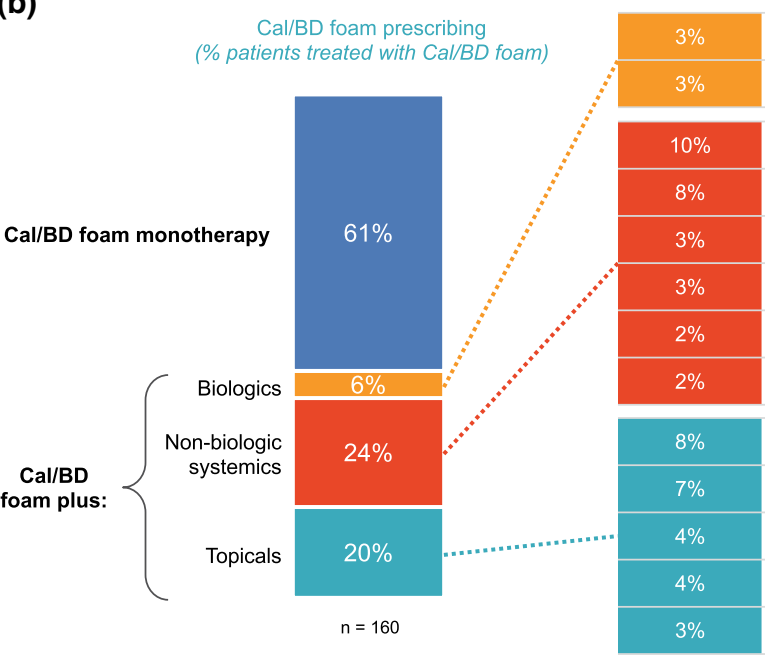

Fig. 3 Treatments with which Cal/BD foam is used as an adjunct (when prescribed as part of multi-therapy). a Germany; b Spain; c UK. Cal/BD foam used as part of combination treatment (e.g., biologic, non-biologic systemic, and topical therapy) in $42 \%$ of patients. Multiple topical therapy treatments were not necessarily used on the same area. A patient may be included in two or more

\section{HCP Preference in Specific Patient Subpopulations}

The most prescribed topical therapy for use as an add-on to biologics in beyond-mild patients
Anti-TNF biologics

Anti IL-23/Anti IL-12

Methotrexate

Acitretin

Apremilast

Cyclosporine

Dimethyl fumarate

Phototherapy

Betamethasone diproprionate and salicylic acid fixed dose combination

Calcipotriol and betamethasone dipropionate gel

Clobetasol propionate ointment

Betamethasone

Other prescribed topical treatments

groups in the combination therapy groups; hence, the total percentage in the monotherapy and combination groups may be greater than $100 \%$. Treatments prescribed to more than $1 \%$ of patients treated with $\mathrm{Cal} / \mathrm{BD}$ foam shown. $\mathrm{Cal} / \mathrm{BD}$ calcipotriol and betamethasone dipropionate, IL interleukin, TNF tumor necrosis factor

was Cal/BD foam (by $68 \%$ of specialists). HCPs cited overall efficacy (83\%), tolerability (61\%), and improvement of patients' QoL (57\%) as their reasons. Although the number of beyondmild patients who were prescribed biologic 


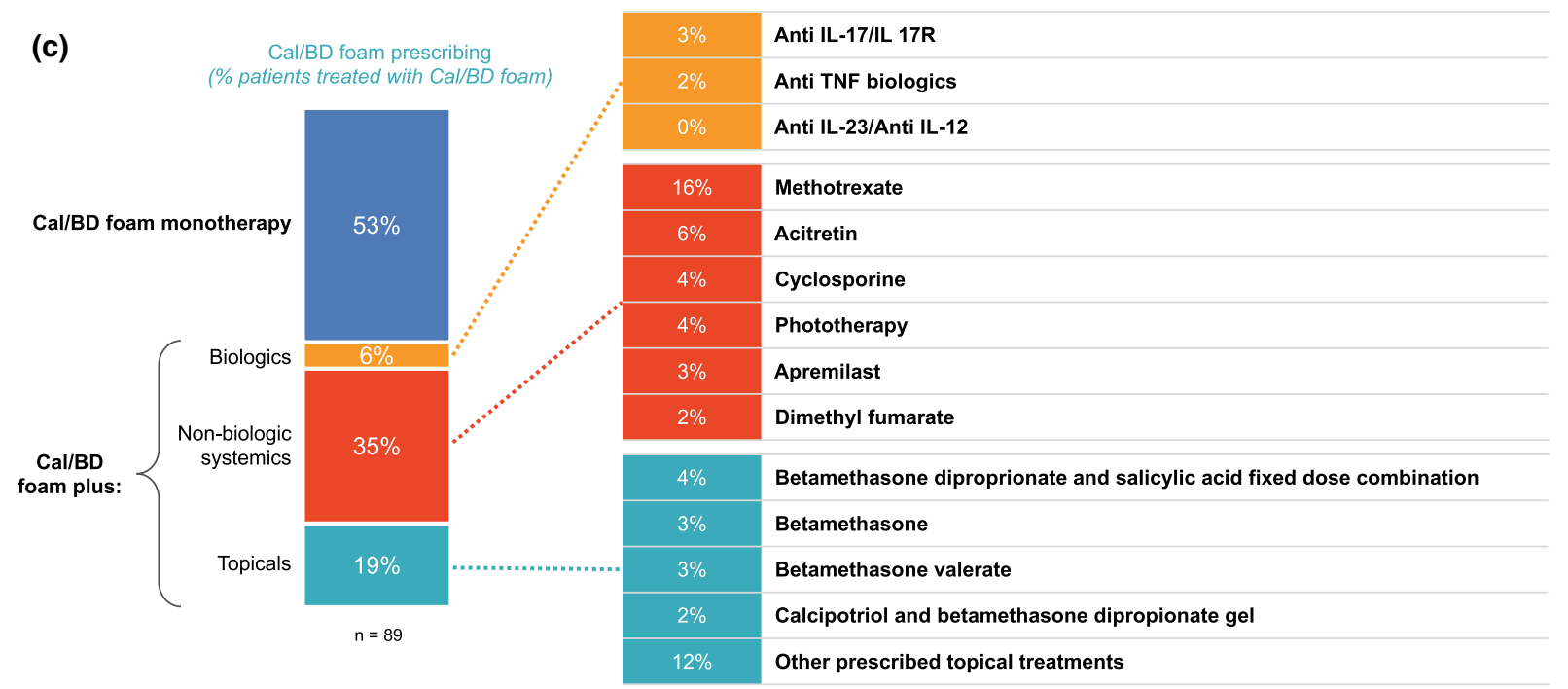

Fig. 3 continued

therapy was relatively small (36\%), almost all specialists (97\%) said they prescribed biologics in beyond-mild psoriasis.

Specialists also prescribed topicals as an alternative to non-biologic systemic therapy in certain situations, such as patient preference (51\%), contraindication (50\%), and to limit side effects (26\%) (Fig. 4). In these situations, Cal/BD foam was prescribed by $68 \%$ of specialists; the reasons given were overall efficacy $(80 \%)$, tolerability (76\%), and improvement of patients' QoL (54\%).

\section{Cal/BD Foam as a Topical Treatment Before Systemic Treatment}

In patients where $\mathrm{Cal} / \mathrm{BD}$ foam-containing regimens were considered effective, specialists reported they were able to postpone initiation of systemic therapy for $21 \%$ of their patients.

\section{Stated frequency of topical prescribing: Non-biologic statements (\% of HCPS)}

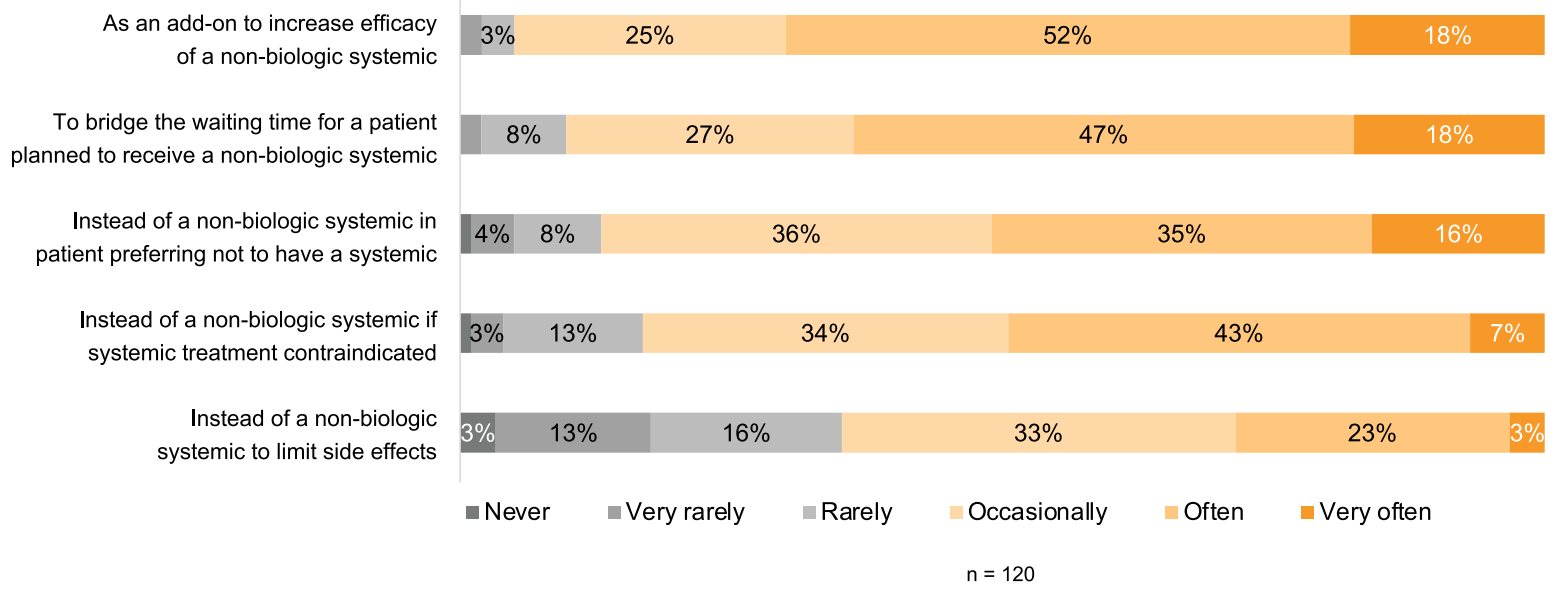

Fig. 4 Healthcare professional reasons for prescribing topical therapies in relation to non-biologic systemic therapies. Values of $2 \%$ or below are not shown 


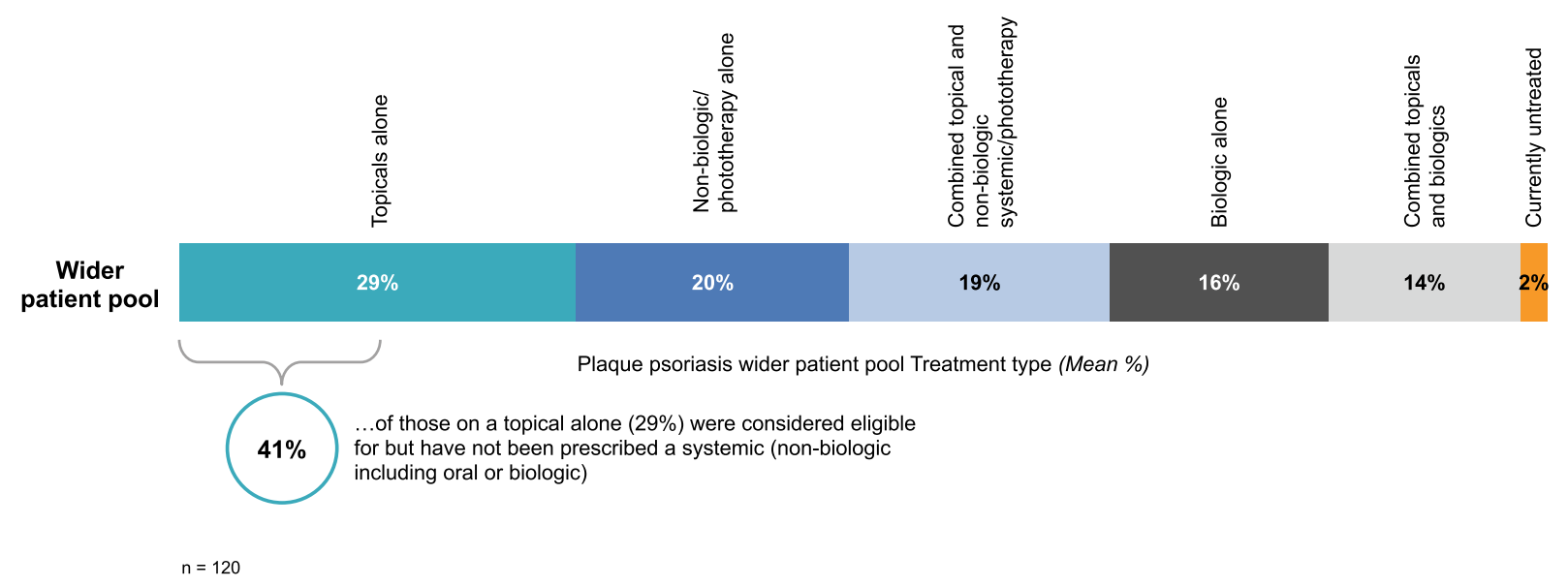

Fig. 5 Patients eligible for systemic therapies but maintained on topical therapies

Improved QoL (59\%) and PASI scores (56\%) were considered to be the main indicators of efficacy.

The majority (78\%) of HCPs believed that the advent of more efficacious topical treatments resulted in a wider pool of patients who can be treated adequately with topical treatments alone. This finding was consistent with the $59 \%$ of HCPs preferring to keep their beyond-mild patients on topical treatments alone when it was an option to do so. Moreover, among their wider patient pool, $41 \%$ of patients currently on topical monotherapy were considered eligible for, but had not been prescribed, a systemic therapy (Fig. 5).

\section{DISCUSSION}

Cal/BD foam is a treatment for plaque psoriasis that has been on the European market for 5 years and which appears to have greater efficacy than Cal/BD ointment or gel formulations, including for moderate-to-severe psoriasis [17]. This research gathered insights from HCP experience on the use of Cal/BD foam for the treatment of beyond-mild psoriasis. While there are real-world data published in this area [17-19], there is a need to share more clinical experience of $\mathrm{Cal} / \mathrm{BD}$ foam in the beyond-mild segment.

Our research indicates that prescribers of $\mathrm{Cal} / \mathrm{BD}$ foam most commonly used this treatment as monotherapy, and as adjunctive therapy with non-biologic systemic therapies for beyond-mild patients. It was used to a lesser extent with other topical treatments in multitherapy and as an adjunct to biologic systemic therapies.

\section{Cal/BD Foam as a Monotherapy in the Beyond-Mild Setting}

Our research found evidence of monotherapy use in the beyond-mild segment treated with $\mathrm{Cal} / \mathrm{BD}$ foam-an observation that is aligned with findings from recent clinical studies, confirming the efficacy of Cal/BD foam in the treatment of beyond-mild plaque psoriasis. PSO-ABLE was a phase III, randomized study assessing the response to $\mathrm{Cal} / \mathrm{BD}$ foam and $\mathrm{Cal} / \mathrm{BD}$ gel in patients with mild-to-severe psoriasis involving less than $30 \% \mathrm{BSA}$, in which $\mathrm{Cal} / \mathrm{BD}$ foam demonstrated superior efficacy at week 4 versus Cal/BD gel at week 8 [15]. Furthermore, in a post hoc subgroup analysis of this study, Paul et al. [15] investigated the efficacy of either Cal/BD foam or gel in a subgroup of patients with moderate-to-severe psoriasis (BSA $\geq 10 \% /$ PASI [excluding head]; modified PASI $[\mathrm{mPASI}]>10 / \mathrm{DLQI}>10$ ) who were eligible for systemic therapy. Study results demonstrated an overall reduction (64\%) in mPASI from baseline to week 12 in patients treated with Cal/BD foam (monotherapy) [15], leading the authors to hypothesize that topical 
treatment might be a significant cost-saving alternative to systemic therapy. In addition to these study data, a matching-adjusted indirect comparison of data from four pooled clinical trials of Cal/BD foam with those from clinical trials of non-biologic systemic therapies, such as methotrexate, found that Cal/BD foam offered superior outcomes [20].

Such findings are consistent with those from real-world studies of $\mathrm{Cal} / \mathrm{BD}$ foam use. A prospective, observational study of $\mathrm{Cal} / \mathrm{BD}$ foam examined the efficacy and tolerability in patients $(N=410)$ with plaque psoriasis ranging from mild to severe, under daily practice conditions. After 4 weeks of treatment, $43.33 \%$ of patients with severe psoriasis (Investigator's Global Assessment [IGA] score $=4$ ) met the study criteria for treatment success, defined as clear/almost clear lesions and at least a two-step improvement in IGA [18]. In another prospective, observational study, 4 weeks of treatment with Cal/BD foam not only provided a significant improvement in PASI and Physician Global Assessment (PGA) indices ( $p<0.001$ for both) but was also associated with a significant reduction in mean pain intensity score $(p<0.001)$ in 75 patients with moderate-tosevere plaque psoriasis [19]. In addition, a review by Pinter et al. described a case study of a patient with moderate-to-severe psoriasis (PASI 12, DLQI 14, BSA 13\%), whose treatment history included an unsatisfactory response to methotrexate during pre-screening for biologic therapy. Following treatment with $\mathrm{Cal} / \mathrm{BD}$ foam (monotherapy), the patient reached PASI 75 improvement, along with complete resolution of his itching. The patient was then started on biologic treatment [14].

\section{Cal/BD Foam in Combination Therapy with Biologic Systemic Treatments}

Among patients receiving Cal/BD foam in the beyond-mild segment, a low proportion received it in combination with biologics. This finding is likely to be due to patients with more severe psoriasis (PASI $>20$ or BSA $>30 \%$ ) who are typically treated with biologics being excluded from the study. Moreover, since biologic therapy is the least frequently prescribed in the beyond-mild segment, it is not surprising to observe that use of $\mathrm{Cal} / \mathrm{BD}$ foam as adjunctive therapy to biologics was low. Therefore, as this research evaluated patients using Cal/BD foam (and not patients using biologics), it should be noted that this finding is not a reflection of the overall use of $\mathrm{Cal} / \mathrm{BD}$ foam in the segment of patients typically treated with biologics. However, Cal/BD foam has been shown to improve treatment outcomes in plaque psoriasis when used as an adjunct to biologics. For example, enhanced outcomes have been observed, in an open-label prospective study, among patients with chronic plaquetype psoriasis with an inadequate response to biologics following the addition of Cal/BD foam to their treatment regimen [21].

\section{HCP Preference in Specific Patient Subpopulations}

There was some evidence to support the hypothesis that Cal/BD foam use was favored as an add-on to biologic therapy and as an add-on or alternative to non-biologic systemic therapy. These findings are based on preferences and behaviors observed in this research. Although there is, for instance, evidence showing Cal/BD foam in combination with apremilast to be well tolerated and associated with improvements in PASI, PGA, and Dermatology Life Quality Index (DLQI) scores [22], further research is needed to better understand HCP preference for $\mathrm{Cal} / \mathrm{BD}$ foam in specific populations, including as an add-on to non-biologic systemic therapy.

\section{Cal/BD Foam as a Topical Treatment Before Systemic Treatment}

There was limited evidence from the research to support the hypothesis that Cal/BD foam can prolong the use of topical treatment before systemic treatment. HCPs indicated that they believed the availability of more efficacious topical therapies resulted in a wider patient pool that can be treated with such therapies. This finding is consistent with that seen in the PSOABLE study subgroup analysis (as mentioned 
earlier), in which the study authors concluded, on the basis of the observed efficacy of Cal/BD foam in moderate-to-severe psoriasis, that "Cal/BD foam can be considered as a treatment option in some patients with moderate-to-severe psoriasis who are potential candidates for systemic therapy" [15]. A recent paper collecting the opinion of an Italian expert panel concluded that $\mathrm{Cal} / \mathrm{BD}$ foam is the topical therapeutic alternative that can change psoriasis treatment approach for both mild and moderate psoriasis [17].

Therefore, while it is our understanding that HCPs do not wish to prolong or to add hurdles to systemic treatment, our findings suggest that Cal/BD foam has the potential to expand the pool of patients with beyond-mild psoriasis who are treated adequately without systemic therapy. This type of prescribing behavior may result in a cost-benefit advantage. It is, however, important to note that when the need for systemic therapies for a patient has been identified, the initiation of such treatments should not be postponed.

\section{Strengths and Limitations}

The questionnaire used in this research was carefully designed and tested in a pilot phase before fieldwork, to ensure that the questions, language, and tasks were clear, and that appropriate vocabulary was used. Nevertheless, there is a reliance on the HCP (and hence susceptibility to human error) to correctly complete the questionnaire. With this in mind, the questionnaire was designed to prompt participants if the incorrect patient was selected.

The highly specific population evaluated here allowed for detailed and specific examination of Cal/BD foam use in this patient segment. While such a population was required to answer the specific objectives of the research, only including patients who had been prescribed Cal/BD foam could be perceived as a limitation. As such, the results here cannot be generalized to other products (but such generalization was not an objective of the research).

The use of biologic therapies may have been underestimated in this sample of patients, since data were derived from GPwSI, who are unable to prescribe these agents, and from dermatologists, who may not necessarily work in psoriasis referral centers. However, it should be noted that patients with more severe psoriasis (PASI $>20$ or BSA $>30 \%$ ) were not the focus of this study and so the proportion of patients receiving biologic therapy was not expected to be high.

The participation of HCPs from Germany, Spain, and the UK provided insights and allowed comparison of Cal/BD foam use within these three countries. The HCP selection criteria employed in this study, requiring participants to have written at least 10 prescriptions for Cal/ BD foam within the last 3 months, could be perceived as another limitation. However, given that $88 \%$ of dermatologists and $74 \%$ of GPwSI were eligible to participate, the HCP selection criteria did not jeopardize the representative aspect of the sample in favor of prescribers of $\mathrm{Cal} / \mathrm{BD}$ foam. That said, this sample of three countries, and the sampling method used (that focused on a subpopulation of HCPs), mean that the results can only very carefully be extrapolated to patients with distinct treatment patterns and to the behaviors of the European market as a whole. While a key strength of the study is that it provides an insight into the realworld efficacy of Cal/BD foam, this approach also introduces the variables and biases inherent to real-world studies, and mentioned above, that may be considered as limitations.

\section{CONCLUSIONS}

New topical options, such as Cal/BD foam, have shown excellent results in patients with mildto-moderate psoriasis, thus reducing the use of the various systemic options $[14,15,18,21]$. This research investigated the current use of $\mathrm{Cal} / \mathrm{BD}$ foam in the moderate-to-severe patient segment in clinical practice and clearly shows a high use of monotherapy when using Cal/BD foam to treat beyond-mild psoriasis. In addition, Cal/BD foam use as adjunctive therapy, with non-biologic systemic treatments and, to a lesser extent, biologic systemic therapies, was noted. 
The HCPs reported high levels of satisfaction when using Cal/BD foam, either as monotherapy or adjunctive therapy. Moreover, HCPs in this research believed that the availability of increasingly efficacious topical treatments has widened the pool of patients eligible for such treatment. Giving patients the choice of an alternative treatment for psoriasis supports a "patient-centered" approach, which may enhance treatment adherence [23].

The potential cost-benefit implications when using Cal/BD foam as a treatment for beyond-mild psoriasis are yet to be determined. It has been suggested that the use of $\mathrm{Cal} / \mathrm{BD}$ foam as opposed to systemic treatment may result in cost savings [24]. Given that treatment cost can impact an HCP's choice of treatment, further insights from real-world experience would help determine the most effective use of $\mathrm{Cal} / \mathrm{BD}$ foam as a treatment option in this patient segment.

\section{ACKNOWLEDGEMENTS}

Funding. The market research cited in this paper and the journal's Rapid Service Fee was funded by LEO Pharma A/S Ballerup, Denmark. Medical Writing Assistance: Medical writing support was provided by Rudy Sarmah, BSc, and Anthony Zucker, PhD, for Cello Health Communications. The support was funded by LEO Pharma A/S Ballerup, Denmark.

Authorship. All named authors meet the International Committee of Medical Journal Editors (ICMJE) criteria for authorship for this article, take responsibility for the integrity of the work as a whole, and have given their approval for this version to be published.

Disclosures. Roland Aschoff reports personal fees from Biofrontera and LEO Pharma for participation in advisory boards and lectures, and personal fees from AbbVie, Galderma, Mylan, and Sanofi for lecture participation. Antonio Martorell has received personal fees and non-financial support from AbbVie, Janssen, UCB, and Lilly, and personal fees from
Novartis, LEO Pharma, and MSD. Tobias Anger is a full-time employee of LEO Pharma as a Medical Advisor. Diane Chayer is a full-time employee of LEO Pharma as Head of Commercial Insights. Anthony Bewley reports a research grant from the European Academy of Dermatology Venereology for Practical Psychodermatology; ad hoc consultancy and travel grants from Almirall, Janssen and LEO Pharma; and consultancy fees from AbbVie, Almirall, Celgene, Galderma, Janssen, LEO Pharma, Lilly, Novartis, Sanofi, UCB; he is also Treasurer and a member of guidelines committees of the British Association of Dermatology, Secretary of the European Society for Dermatology and Psychiatry, Chair of the All-Party Parliamentary Group on Skin, and acts as an Advisor for the Psoriasis Association, Changing Faces, Ichthyosis Support Group, and the National Eczema Society.

Compliance with Ethics Guidelines. The names and other identifying information about the specialists and the patient data they provided were confidential, and not shared with the study sponsor. Informed consent was obtained from all HCPs, and the research was conducted in accordance with guidelines/codes of conduct regarding anonymity and confidentiality from the Association of the British Pharmaceutical Industry, the European Pharmaceutical Market Research Association, the European Society for Opinion and Marketing Research, the Market Research Society, the British Healthcare Business Intelligence Association, and the European General Data Protection Regulation. Specialists were also informed about the compensation for their time, that all patient cases were anonymized, and that only adults were to be included. As all patient cases were anonymized, no personal data were retrospectively collected from available patient charts, and no written informed consent was required from patients. As such, approval by an ethics committee or institutional review board was not required.

Data Availability. The datasets generated and/or analyzed during the current study will be made available on reasonable request made to the authors. 
Open Access. This article is licensed under a Creative Commons Attribution-NonCommercial 4.0 International License, which permits any non-commercial use, sharing, adaptation, distribution and reproduction in any medium or format, as long as you give appropriate credit to the original author(s) and the source, provide a link to the Creative Commons licence, and indicate if changes were made. The images or other third party material in this article are included in the article's Creative Commons licence, unless indicated otherwise in a credit line to the material. If material is not included in the article's Creative Commons licence and your intended use is not permitted by statutory regulation or exceeds the permitted use, you will need to obtain permission directly from the copyright holder. To view a copy of this licence, visit http://creativecommons.org/licenses/by$\mathrm{nc} / 4.0 /$.

\section{REFERENCES}

1. Parisi R, Symmons DPM, Griffiths CEM, Ashcroft DM. Global epidemiology of psoriasis: a systematic review of incidence and prevalence. J Invest Dermatol. 2013;133:377-85.

2. Sarac G, Koca TT, Baglan T. A brief summary of clinical types of psoriasis. North Clin Istanb. 2016;3:79-82.

3. Griffiths CEM, Jo S-J, Naldi L, et al. A multidimensional assessment of the burden of psoriasis: results from a multinational dermatologist and patient survey. Br J Dermatol. 2018;179:173-81.

4. Kimball AB, Jacobson C, Weiss S, Vreeland MG, Wu Y. The psychosocial burden of psoriasis. Am J Clin Dermatol. 2005;6:383-92.

5. Knuckles MLF, Levi E, Soung J. Defining and treating moderate plaque psoriasis: a dermatologist survey. J Dermatol Treat. 2018;29:658-63.

6. Gisondi P, Del Giglio M, Girolomoni G. Treatment approaches to moderate to severe psoriasis. Int J Mol Sci. 2017;18:2427.

7. Menter A, Korman NJ, Elmets CA, et al. Guidelines of care for the management of psoriasis and psoriatic arthritis. Section 3. Guidelines of care for the management and treatment of psoriasis with topical therapies. J Am Acad Dermatol. 2009;60:643-59.
8. Weigle N, McBane S. Psoriasis. Am Fam Physician. 2013;87:626-33.

9. Mrowietz U, Kragballe K, Reich K, et al. Definition of treatment goals for moderate to severe psoriasis: a European consensus. Arch Dermatol Res. 2011;303:1-10.

10. National Institute for Health and Excellence. NICE pathways. Topical therapy for psoriasis. https:// pathways.nice.org.uk/pathways/psoriasis. 2019. Accessed 26 Feb 2021.

11. Armstrong AW, Villanueva Quintero DG, Echeverría CM, Gu Y, Karunaratne M, Reyes Servín O. Body region involvement and quality of life in psoriasis: analysis of a randomized controlled trial of adalimumab. Am J Clin Dermatol. 2016;17: 691-9.

12. Afifi T, de Gannes G, Huang C, Zhou Y. Topical therapies for psoriasis: evidence-based review. Can Fam Physician. 2005;51:519-25.

13. Leo Laboratories Limited. Enstilar 50 micrograms/g $+0.5 \mathrm{mg} / \mathrm{g}$ cutaneous foam. Summary of product characteristics. https://www.medicines.org.uk/emc/ medicine/31833\#gref. 2020. Accessed 26 Feb 2021.

14. Pinter A, Thormann H, Angeletti F, Jalili A. Calcipotriol/betamethasone dipropionate aerosol foam for the treatment of psoriasis vulgaris: case series and review of the literature. Clin Cosmet Investig Dermatol. 2018;11:451-9.

15. Paul C, Leonardi C, Menter A, et al. Calcipotriol plus betamethasone dipropionate aerosol foam in patients with moderate-to-severe psoriasis: subgroup analysis of the PSO-ABLE study. Am J Clin Dermatol. 2017;18:405-11.

16. Koo J, Tyring S, Werschler WP, et al. Superior efficacy of calcipotriene and betamethasone dipropionate aerosol foam versus ointment in patients with psoriasis vulgaris-a randomized phase II study. J Dermatol Treat. 2016;27:120-7.

17. Megna M, Cinelli E, Camela E, Fabbrocini G. Calcipotriol/betamethasone dipropionate formulations for psoriasis: an overview of the options and efficacy data. Expert Rev Clin Immunol. 2020;16: 599-620.

18. Gerdes S, Krakor M, Anger T, Hutt HJ, Körber A. Prospective, observational, non-interventional, multicentre study on the efficacy and tolerability of a new calcipotriol/betamethasone aerosol foam $\left(\right.$ Enstilar $\left.^{\circledR}\right)$ in patients with plaque psoriasis under daily practice conditions. Dermatology. 2017;233: 425-34. 
19. Gallo L, Megna M, Cirillo T, et al. Psoriasis and skin pain: real-life effectiveness of calcipotriol plus betamethasone dipropionate in aerosol foam formulation. J Eur Acad Dermatol Venereol. 2019;33: 1312-15.

20. Bewley AP, Shear NH, Calzavara-Pinton PG, Hansen JB, Nyeland ME, Signorovitch J. Calcipotriol plus betamethasone dipropionate aerosol foam vs. apremilast, methotrexate, acitretin or fumaric acid esters for the treatment of plaque psoriasis: a matching-adjusted indirect comparison. J Eur Acad Dermatol Venereol. 2019;33:1107-15.

21. Bagel J, Zapata J, Nelson E. A prospective, openlabel study evaluating adjunctive calcipotriene $0.005 \%$ /betamethasone dipropionate $0.064 \%$ foam in psoriasis patients with inadequate response to biologic therapy. J Drugs Dermatol. 2018;17: 845-50.
22. Kircik L, Schlesinger TE, Tanghetti E. Efficacy and safety of calcipotriene $0.005 \%$ /betamethasone dipropionate $0.064 \%$ foam with apremilast for moderate plaque psoriasis. J Drugs Dermatol. 2020;19:874-80.

23. Lowes R. Patient-centered care for better patient adherence. Fam Pract Manag. 1998;5:46-57.

24. Becla L, Carrascosa JM, Antunes JF, Bewley AP, Delgado Perala M, Charrua MF. Cost per responder analysis of calcipotriol plus betamethasone dipropionate aerosol foam versus non-biologic systemic therapy in the treatment of moderate to severe plaque psoriasis. Value Health. 2018;21(Suppl 3): S425 (Abstract PSS16). 